\title{
Investigation of Noncovalent Interaction Applications in the Organic Chemistry Teaching
}

\author{
Zhi-min Huang \\ School of Environment and Chem. Eng. \\ Nanchang Hangkong University \\ Nanchang, P.R. China
}

\author{
Yong-fen Tong \\ School of Environment and Chem. Eng. \\ Nanchang Hangkong University \\ Nanchang, P.R. China
}

\begin{abstract}
The organic chemical reaction is a process of covalent bond breaking and forming. A number of discussions and research during organic chemistry teaching have been given to the properties and applications of various organic compounds' covalent bonds. Compared with covalent bond interaction, noncovalent interaction bond is weaker. Nevertheless, noncovalent interactions widely exist in the individual compounds and among the compounds, and are extensively applied in the organic functional materials. This paper has investigated noncovalent interaction's effect on physical properties of organic compound, organic compounds' and their intermediates' stability, reaction activity, acidity and reaction direction of organic compounds and applications of organic compound properties from a unique respective. It is our expectation that effect of noncovalent interaction will be given more considerations in the organic chemistry teaching and learning, and our practices provide a new respective to the organic chemistry innovative ideas.
\end{abstract}

Keywords-Noncovalent interaction; Organic chemical teaching; Application; Investigation

\section{INTRODUCTION}

The Chinese characters for the "Chemistry" mean the "Science of Change" if understood literally only. The chemistry is, as same as physics, one of science basics. The properties of material, which are presented in the event of chemical changes, are defined as the chemical properties, such as flammability, instability, acidity, alkalinit, oxidizability and reducibility, etc. Chemical changes may be given an easily understood expression: a type of changes in which there are other substances generated in the process of experiment. The properties of materials, which can be presented without a requirement of chemical change, are defined as physical properties, such as the color, state, smell, melting point, boiling point, density and solubility, etc. The nature of the chemical reaction is determined by the movement and restructuring of extra-nucleus electrons. In the process of chemical analysis, atomic structure and pattern of extranuclear electrons are those important factors, which determine the properties of the elements and materials. The chemical bond is generally defined as a strong interaction between adjacent two or more atoms (or ions) within the molecule and crystal of purified material. The chemical bond is divided into ionic bond, covalent bond and metallic bond.

The organic chemistry is a science field which studies the constituent, structure, properties and law of change of organic compounds, and also an important branch of chemistry. The carbon elements are included in the constituent of all organic compounds, such as ethyl alcohol, acetic acid and sucrose. Extra-nucleus electron pattern of carbon element determine that all organic compounds are combined with covalent bonds.

The covalent bonds have characteristics that two or more atoms jointly utilize all of their electrons around outer level orbitals. Given the perfect saturation condition of electron, the covalent bonds can establish stable and solid chemical structure. The organic chemical reaction is a process, in which old covalent bonds are broken while new covalent bonds are formed. Therefore, a number of discussions in the organic chemistry teaching have been given to the length, angle, and energy, polar and break mode of various organic compounds' covalent bonds. This is also the main contents of organic chemistry teaching.

In addition to strong covalent bond interactions of atoms within the molecule in the organic compounds, there exist noncovalent interactions. Compared with covalent bond interactions, noncovalent interactions are classified as weak interactions, mainly including Van der Waals interaction, electrostatic gravitation, hydrogen bond interaction, $\pi$ interaction, host-guest system recognition and hydrophobic interaction, etc. Although their interactions range from several KJ.mol-1 to dozens of KJ.mol ${ }^{-1}$, much less than hundreds to thousands of KJ. mol ${ }^{-1}$ of covalent interactions, noncovalent interactions still have an important effect on the physical properties, chemical properties and biological properties of organic compounds.

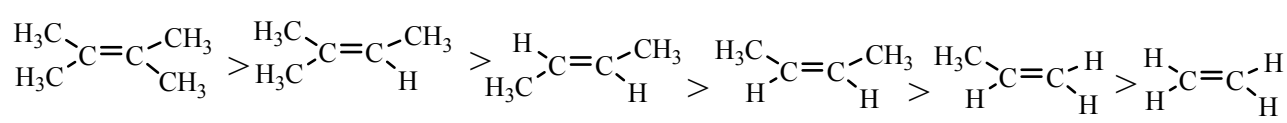

When learning organic chemistry analysis and judging the properties of organic compound, the students often focused on the atomic covalent interactions of organic compound, but have not considered enough about noncovalent interactions among the nonbonding atoms. This is easily to cause misunderstanding about properties of material, direction of reaction and application prospective. The following will 
investigate the applications of noncovalent interaction in organic chemistry.

\section{EfFects on The Physical Properties of Organic COMPOUNDS}

Some of physical properties, such as state, melting point, boiling point and solubility of organic compounds, are determined by the noncovalent interactions between organic compounds, or between organic compounds and other compounds. The greater covalent interaction is, the greater interaction between molecules is, and the higher melting point and boiling point are. Take the oxidation of approximate molecular weights. The order of boiling points from high to low is: carboxylic acid >alcohol >aldehyde >ether. This is because that carboxylic compound may form two molecular hydrogen bonds, alcohol substance may form one molecular hydrogen bond, and aldehyde substance can't form hydrogen bond at all but has an induction effect, while the molecular interaction of ether substance is the weakest.

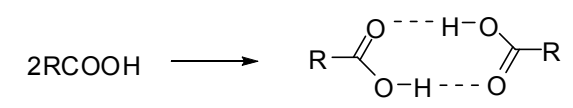

Fig. 1. Dipolymer Structure of Acetic Acid

Butanol and ether are the isomers. Their boiling points are $117.3^{\circ} \mathrm{C}$ and $34.5^{\circ} \mathrm{C}$ respectively, while their solutions in the water are approximately the same $8 \mathrm{~g} .100 \mathrm{~g}-1$.O-nitropheno and p-nitropheno may be separated and purified with steam distillation method. The separation and purification of other substances with similar structure may be decided based on their property that noncovalent interactions - hydrogen bonds, exist between these substances, or between these substances and water.

\section{EFFECTS ON THE STABILITY OF ORGANIC COMPOUNDS AND INTERMEDIATES}

When discussing the stability of olefin, the following conclusion may be obtained based on the data analysis of heat of hydrogenation of olefin.

The more the hydrogen atoms of ethylene molecule are substituted by the methyl, the more stable the molecule is. Trans 2-butylene is more stable than cis 2-butylene.The reason is: the electrons on the $\sigma$ orbital of methyl $\mathrm{C}-\mathrm{H}$ may transfer to the $\pi$ orbital of adjacent $\mathrm{C}=\mathrm{C}$, which will result in an interaction of charge delocalization. This interaction of charge delocalization is a noncovalent interaction. The more the methyls are, and the more the $\sigma$ electrons presenting in the charge delocalization are, therefore the more stable the olefins are.

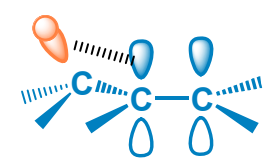

Fig. 2. Propylene $\sigma-\pi$ noncovalent interaction

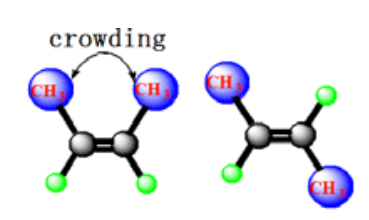

Fig. 3. Structure of 2-butylene

It is shown from Fig. 3 that the positions of two methyls of cis 2-butylene are quite near, which, therefore, results in a large noncovalent interaction, ie., Van der Waals repelling interaction, between $\sigma$ electrons of two methyls. The structure stability of cis 2-butylene is weaker than that of trans 2butylene.

The stability order of alkyl group $\mathrm{C}+$ and $\mathrm{C}$, may be explained by using the charge delocalization interaction, which is produced by transferring the electron of alkyl $\mathrm{C}-\mathrm{H}$ from the occupied $\sigma$ orbital to adjacent vacant $\mathrm{p}$ orbital.<smiles>C[CH+]CCCC(C)C</smiles>

Fig. 4. Stability order of alkyl group $\mathrm{C}^{+}$<smiles>C1CCCCC1</smiles><smiles>[H][R]C1CCCCC1</smiles>

Fig. 5. Conformation of ethane

The facts that stability of intersection conformation of alkyl is larger than that of superimposed conformation of alkyl, stability of $\beta$ - naphthalene sulfonic acid is larger than that of $\alpha$ naphthalene sulfonic acid, may be explained by using the nonbonding Van der Waals repelling interaction between atoms.[1]

\section{EFFECTS ON THE EXTENT OF REACTION ACTIVITY OF ORGANIC COMPOUNDS}

The organic chemical reaction is a process of covalent bond breaking and forming, but it is very difficult to explain the activity of some organic reactions based on the energy grade of covalent bond. For example, when vinyl chloride and allyl chloride react respectively with alcoholic solution of silver nitrate, nitrate ester is generated with the same single bond reaction. But the reaction with allyl chloride is easier than with vinyl chloride. Under ambient temperature, white silver chloride deposit can be generated with allyl chloride very quickly, while it is very difficult to react with vinyl chloride even heated for several hours.

$\mathrm{CH}_{2}=\mathrm{CH}-\mathrm{Cl}+\mathrm{AgNO}_{3} \stackrel{\text { alc. }}{\longrightarrow} \mathrm{CH}_{2}=\mathrm{CH}-\mathrm{ONO}_{2}+\mathrm{AgCl} \downarrow$
$\mathrm{CH}_{2}=\mathrm{CH}-\mathrm{CH}_{2}-\mathrm{Cl}+\mathrm{AgNO}_{3} \stackrel{\text { alc. }}{\longrightarrow} \mathrm{CH}_{2}=\mathrm{CH}-\mathrm{CH}_{2}-\mathrm{ONO}_{2}+\mathrm{AgCl} \downarrow$ 

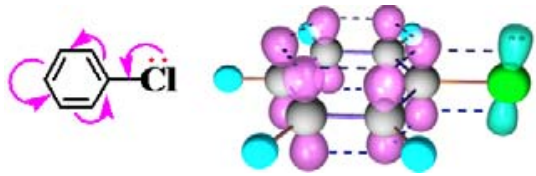

Fig. 6. $\mathrm{p}-\pi$ noncovalent interaction of vinyl chloride

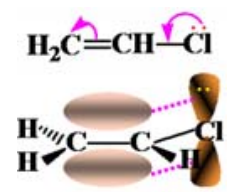

Fig. 7. $\mathrm{p}-\pi$ noncovalent interaction of chlorobenzene

This is because those electrons on the outer level orbital $p$ of chloride may transfer to adjacent orbital $\pi$ of ethyl $\mathrm{C}=\mathrm{C}$, then noncovalent interaction is formed between $\mathrm{C}-\mathrm{Cl}$. This makes $\mathrm{C}-\mathrm{Cl}$ bonded much stronger, bond length shorter and molecule polar weaker. It is why the reaction is difficult to be performed. The fact that reaction activity of $\mathrm{Cl}$ in the chlorobenzene is weaker than that of $\mathrm{Cl}$ in the benzyl chloride may be explained in the same way.

\section{EFFECTS ON THE EXTENT OF ACIDITY OF ORGANIC COMPOUNDS}

The organic compound acidities are obtained by comparing with the concentration value $\mathrm{pKa}$ of ionized hydrogen ions. It means that acidity of organic compound depends on the strength of $\mathrm{O}-\mathrm{H}$ or $\mathrm{C}-\mathrm{H}$ bond in the organic compound molecule. The greater the bonding energy between atoms is, the weaker the acidity is $\mathrm{O}-\mathrm{H}$ and $\mathrm{C}-\mathrm{H}$ bonding energies are subject to the effect of other polar covalent interaction bonded with $\mathrm{O}$ and $\mathrm{C}$ heavily. The breaking of $\mathrm{O}-\mathrm{H}$ is also subject to the effect of noncovalent interaction in an appropriate environment. Take the cooperation of $\mathrm{o}, \mathrm{m}, \mathrm{p}$-nitrophenol acidity order. The effect strength order of nitro- vs $\mathrm{O}-\mathrm{H}$ bonding energy covalent interaction is: $0>m>>p$. But actual result is as shown in the Fig. 8: $p>0>m$. This is because $\pi-\pi$ noncovalent interaction exists between the $o, p$ nitro- and benzene ring, which disperses the density of electron cloud of oxygen, makes $\mathrm{O}-\mathrm{H}$ bonding energy strength weak, and therefore makes $o$ and $p$ position acidity greater than that of $m$ position. Furthermore, noncovalent interaction between oposition $\mathrm{O}-\mathrm{H}$ and oxygen in the nitro- causes the breaking of $\mathrm{O}-\mathrm{H}$ much more difficult, so acidity of o-nitrophenol is weaker than that of $\mathrm{p}$ - nitrophenol. The reason that the second ionization of dicarboxylic acid is much weaker than the first ionization may be explained by using the noncovalent interaction similarly[2]. Refer to the Fig. 9.

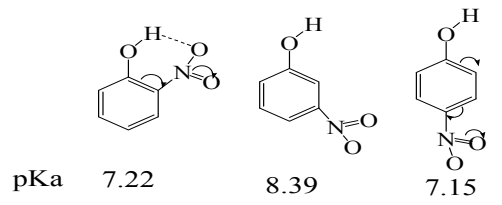

Fig. 8. Acidity comparison of nitrophenol<smiles>O=C1C[CH-]OC(=O)C1</smiles>

Fig. 9. Structure of Malonic acid hydrogen ions

\section{EFFECT ON THE REACTION DIRECTION OF ORGANIC COMPOUNDS}

The property of covalent bond determines the chemical property of material. But, depending on the chemical property difference between homologues, noncovalent interaction resulted from the specific structure may also cause significant effect on the stereoselectivity and regioselectivity of organic compound reaction. Take the elimination reaction of halogenated hydrocarbon. Alkali B:- of cis-attack, together with deionized $\mathrm{X}$-, is easy to generate noncovalent repulsion, which results in higher activation energy of reaction. So, the trans-elimination is the primary reaction.

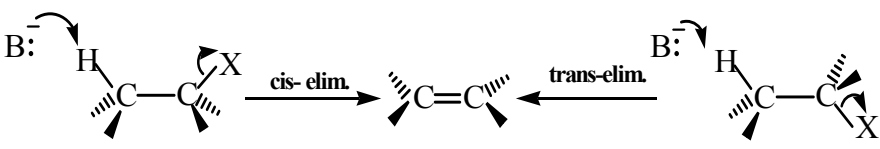

Take the addition reaction between bromethylene, trifluoroethene and $\mathrm{HBr}$. As all of $\mathrm{Br}$ and $\mathrm{CF} 3$ are electron withdrawing groups, they can attract the electron cloud of ethylene towards themselves by way of covalent bond, so the reaction direction of addition compound should be the same. But the resultant reaction direction is at the rightly contrary direction due to the noncovalent interaction of charge delocalization, which results from the transfer of electron $\mathrm{P}$ of reaction intermediate $\mathrm{Br}$ to the vacant orbital $\mathrm{P}$ of adjacent $\mathrm{C}$.
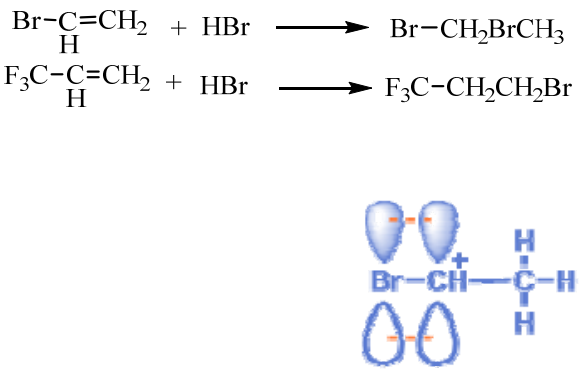

Fig. 10. Structure of intermediate $\mathrm{C}^{+}$

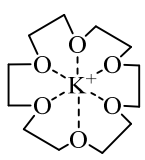

Fig. 11. Structure of 18-crown ether ion $\mathrm{K}$

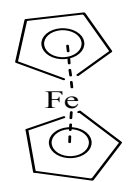

Fig. 12. Structure of ferrocene 


\section{EFFECT ON THE APPLICATION OF ORGANIC COMPOUND PROPERTY}

By using the property of noncovalent interaction resulted from the specific structure of some of organic compounds, it is possible to extend the organic compound chemical reaction to other applications.

Oxygen in the molecules of alkylphenol ethoxylate and fatty alcohol-polyoxyethylene ether, together with water, may form a noncovalent interaction with strong affinity, and is soluble in water. By contrast, the alkyl at another end of structure is apt to form noncovalent interaction with organic compound. Therefore, they have good properties of wash, dispersion, emulsification and humidification, and have been widely used in the industries of textile, coating, cosmetics and fertilizer as well as environment protection.

Crown ether and metallic ions, by way of noncovalent electrostatic interaction, can generate chelate. Refer to the Fig. 11.

Crown ether with various volume of cavity may complex with different metals. By using this property, crown ether may be utilized for the enrichment and separation of metal, and also the phase transfer catalyst. Similarly, upper section hydrophobio cavities of calixarene and neutral molecules, such as chloromethane, benzene and methylbenzene, may generate the inclusion complex with noncovalent interaction. Hydroxy $\mathrm{OH}$ at the bottom section of molecule and positive ions, such as sodium ion, kalium ion, mercury ion and silver ion, may generate the chelates[3] with noncovalent interaction. Calixarene may be used for molecule recognition, electrode materials, mimic enzyme and chemical sensor[4].In addition, ferrocene (Fig. 12) and its derivatives are important organic synthesis intermediates. Due to their unique physical and chemical properties, they have been widely used in the fields such as functional materials and biomedical science.

Self-assembly preparation technology of ultrathin film[5], such as assembly films of polyaniline and polythiophene, is based on that basic structural units, in the event of non-covalent bond interaction, can spontaneously organize or aggregate into a stable structure with specified geometrical conformation. Non covalent bond interaction has been widely applied in these fields such as electroluminescent devices, molecular recognition[6], hydrophobic materials, conductive polymer materials, orderly aggregation assembly, organic molecular devices and emerging organic materials preparation[7].

\section{VIII.CONCLUSION}

Non covalent interactions widely exist in and between organic compounds, and have a significant effect on the properties of organic compound and their applications. Existence of noncovalent interaction should be given enough consideration when learning organic chemistry. When reverserule products, reverse-rule effect, easily formed penta atomic ring and hexa atomic ring products and some products of special property are present, analysis should be made progressively as such that if the covalent interaction exists, what kind of non-covalent interaction it is, and what the reason of interaction formation is. In this way, understanding for organic chemistry can be deepened, ability to analyze and solve problems be improved, which will lay a foundation for becoming innovative talent of organic chemistry.

\section{ACKNOWLEDGMENTS}

This research was financially supported by Jiangxi Education Research Program No: JXJG-15-8-3And NCHU Education Teaching Program No:JY1539

\section{REFERENCES}

[1] Y.M.Li, S.X.Zhao, Y.Y.Wang et al., Organic Chemistry, Second ed., science press., BeiJin, 2014(In Chinese)

[2] W.Q.Zhang, Y.Zhen, R.Ma et al., Organic Chemistry, Fifth ed., Higher Education Press., BeiJin, 2014(In Chinese)

[3] L.X.Zhang, Q.Yang, New Progress of Research in calixarene, J. Applied Chemical Industry 09(2010)

[4] Z.C.Liu, H.Wang, R.Yang et al., Synthesis and

[5] Application of Phosphorus in Calixarenes and Their Complexes , J. Progress in chemistry, 08( 2011) (In Chinese)

[6] X.Y.Shu, R.G.Xie, Synergistic Effect of Non Covalent

[7] Bond in Self-assembly of Supramolecular, J.Chemical Research and Application, 12(2007) (In Chinese)

[8] X.Z.Wang,Y.Q.Chen, X.Z.Chen et al., Synergistic Effect of Non Covalent Bond in Organic Molecular Recog-nition and Aggregatesassembly, J. Progress in chemistry, 03( 2005)(In Chinese)

[7] Y.R.Fan, Z.B.Zhao, W.B.Wan et al., Research Progress of Graphene Non Covalent Functionalization and Its Application J. Chemical Industry and Engineering Progress, 07 (2011) (In Chinese) 